\title{
Empfehlungen der Deutschen Atemwegsliga zur Messung der Atemmuskelfunktion*
}

\author{
Recommendations for Respiratory Muscle Testing
}

Autoren

Institute
H.-J. Kabitz ${ }^{1}$, S. Walterspacher ${ }^{1}$, U. Mellies ${ }^{2}$, C. P. Criée ${ }^{3}$, W. Windisch ${ }^{4}$

${ }^{1}$ Abteilung Pneumologie, Universitätsklinik Freiburg

${ }^{2}$ Abteilung Pädiatrische Pneumologie und Schlafmedizin, Universitätsklinikum Essen

${ }^{3}$ Evangelisches Krankenhaus Göttingen-Weende e.V. Abteilung Pneumologie, Bovenden-Lenglern

${ }^{4}$ Kliniken der Stadt Köln gGmbH - Lungenklinik, Abteilung Pneumologie, Universität Witten/Herdecke, Köln
Bibliografie

DOI http://dx.doi.org/

$10.1055 / \mathrm{s}-0034-1365283$

Online-Publikation: 8.4.2014

Pneumologie 2014; 68: 307-314

(c) Georg Thieme Verlag KG

Stuttgart · New York

ISSN 0934-8387

\section{Korrespondenzadresse}

PD Dr. Hans-Joachim Kabitz

Abteilung Pneumologie,

Universitätsklinik Freiburg,

Killianstraße 5

79106 Freiburg

hans-joachim.kabitz@uniklinik-

freiburg.de

\section{Zusammenfassung}

\section{$\nabla$}

Der atemmuskulären Funktionsdiagnostik kommt aufgrund der klinischen Bedeutung der (Patho-)Physiologie der Atempumpe in weiten Teilen der Pneumologie, Beatmungs- sowie Intensivmedizin eine entscheidende Rolle zu. Neben differenzialdiagnostischen Überlegungen z.B. im prolongierten Weaning und der Quantifizierung der atemmuskulären Einschränkung z.B. bei angeborenen und erworbenen neuromuskulären Erkrankungen, bei COPD oder ventilator-induziertem Zwerchfellschaden ermöglicht diese Diagnostik auch klinische Verlaufskontrollen (z.B. bei spezifischem Atemmuskeltraining).

Grundsätzlich müssen zwei Arten von Techniken unterschieden werden: von der Patientenmitarbeit abhängige versus mitarbeitsunabhängige Verfahren. Als Orientierungshilfe dienen zunächst die weniger komplexen, nicht-invasiven mitarbeitsabhängigen Verfahren. Bei pathologischen Ergebnissen unterhalb des jeweiligen nicht-pathologischen Grenzwertes kommen hiernach die komplexeren, (nicht-invasiven und invasiven) mitarbeitsunabhängigen Techniken inklusive sonografischer Verfahren sowie der Elektromyografie zum Einsatz. Durch dieses Vorgehen lässt sich zwischen Mitarbeitsschwierigkeiten (i.e. falsch-niedrige Messwerte) und manifester Atemmuskelschwäche differenzieren.

Einige Methoden sind komplementär bzw. additiv in ihrer Aussagekraft. Für eine komplette Diagnostik der Atemmuskelfunktion ist daher häufig die Kombination diverser Verfahren notwendig.

Diese Empfehlungen beinhalten Erläuterungen und Handlungsanweisungen zu den diversen mitarbeitsabhängigen und -unabhängigen Verfahren, welche der atemmuskulären Funktionsdiagnostik dienen.

\section{Abstract \\ $\nabla$}

Based on the tremendous impact of impaired respiratory muscle function, tests on their function play a significant role in respiratory and intensive care medicine. Besides differential diagnosing e.g. during prolonged weaning and quantification of impaired respiratory muscle function, e.g. in COPD, neuro-muscular diseases or ventilator-induced diaphragmatic dysfunction, those tests qualify for follow-up assessment, e.g. phrenic nerve lesions or specific respiratory muscle training.

In general, (simple) volitional and (complex) nonvolitional tests are available. Volitional tests aim at screening for potential respiratory muscle impairment, while non-volitional tests - including ultrasound application - are used to further specify low values assessed by volitional tests and to assess complex clinical conditions (e.g. intubated, sedated patients). Several tests are complementary or additive to each other. Complete assessment for respiratory muscle function, therefore, frequently requires the combination of different test regimes.

The current recommendations include in-depth description and practical guidelines for the different tests and approaches to assess respiratory muscle function.

\footnotetext{
* Das Ms. liegt als Monografie „Empfehlungen zur Messung der Atemmuskelfunktion“ in Form einer Einzelpublikation im Dustri Verlag vor: ISBN 978-3-87185-493-4.
} 


\section{Einleitung}

$\nabla$

Die Atemgase werden durch das ventilatorische System an- und abtransportiert [1,2]. Kommt es zu einer Einschränkung der Ventilation, so resultiert hieraus eine alveoläre Hypoventilation mit konsekutiver Hyerkapnie sowie Hypoxämie (i.e. hyperkapnische respiratorische Insuffizienz oder auch ventilatorische Insuffizienz). Die in $\bullet$ Abb. 1 näher beschriebenen neuronalen, muskulären und thorakalen Komponenten stellen in ihrer Gesamtheit die Atempumpe dar und repräsentieren das ventilatorische System [3].

Kommt es zu Einschränkungen der Zwerchfellfunktion oder der nicht-diaphragmalen Inspirationsmuskulatur, so kann hieraus ein myogenes Atempumpversagen resultieren. Entscheidend ist hierbei, inwieweit das Gleichgewicht zwischen der verfügbaren Kapazität der Atempumpe und der ihr auferlegten Last gestört ist $[1,2,4]$. So können Lasterhöhungen wie beispielsweise bei interstitiellen Lungenerkrankungen oder auch Kapazitätsminderungen wie beispielsweise bei einer Muskeldystrophie dieses Gleichgewicht verschieben. Darüber hinaus existieren Krankheitsbilder wie z.B. die COPD, welche beide Komponenten zugleich negativ beeinflussen [3].

Besonderheiten müssen bzgl. der Atemmuskelfunktion bei Kindern beachtet werden: Diese haben einerseits eine geringere Atemmuskelkraft als Erwachsene und andererseits wegen ihres höheren Grundumsatzes und Sauerstoffverbrauchs einen höheren Atemantrieb. Die eingeschränkte Kapazität der Atempumpe ist dafür verantwortlich, dass die Ventilation, insbesondere von Säuglingen und Kleinkindern, bei einer Erhöhung der atemmuskulären Last (z.B. im Rahmen einer Pneumonie bzw. eines Asthmaanfalls) rasch dekompensiert.

Die Messung der Atemmuskelfunktion hat zum Ziel, zum einen zu klären, ob eine atemmuskuläre Einschränkung vorliegt, und wenn ja, in welchem Ausmaß; zum anderen muss geklärt werden, ob und in welchem Maße der Atempumpe eine erhöhte Last auferlegt ist [2,4]. Hierdurch kann eine latente oder manifeste Atemmuskelschwäche diagnostiziert und differenzialdiagnostisch eingegrenzt werden. Des Weiteren existiert die Möglichkeit, klinische Verlaufskontrollen der Atemmuskelfunktion durchzuführen, wie sie z.B. im Rahmen von spezifischem Atemmuskeltraining oder bei neuro-muskulären Erkrankungen zum Einsatz kommen [3].

\section{Messung der Atemmuskelfunktion \\ $\nabla$}

Es sind die Symptome des Patienten, welche initial den Verdacht auf eine mögliche ventilatorische Insuffizienz lenken. Hierzu gehören z. B. Luftnot bei Belastung, im Liegen oder beim Eintauchen des Körpers in Wasser, aber auch eine beschleunigte Ruheatmung, vermehrter Einsatz der Atemhilfsmuskulatur oder paradoxe Atemanstrengungen. Bei fortgeschrittenen Erkrankungen mit chronischer ventilatorischer Insuffizienz kommen klinische Zeichen der Hyperkapnie hinzu - wie morgendliche Kopfschmerzen, Schlafstörungen und periphere Ödeme sowie Zeichen der Rechtsherzinsuffizienz.

Apparativ sollte stets eine kapilläre bzw. arterielle Blutgasanalyse sowie eine Lungenfunktionsdiagnostik durchgeführt werden. Hiernach kommen diverse Verfahren zum Einsatz, welche entweder die atemmuskuläre Kraft oder deren Funktion ermitteln [4,5]. Hierbei müssen mitarbeitsabhängige von -unabhängigen Methoden abgegrenzt werden. Aufgrund der Tatsache, dass eini-

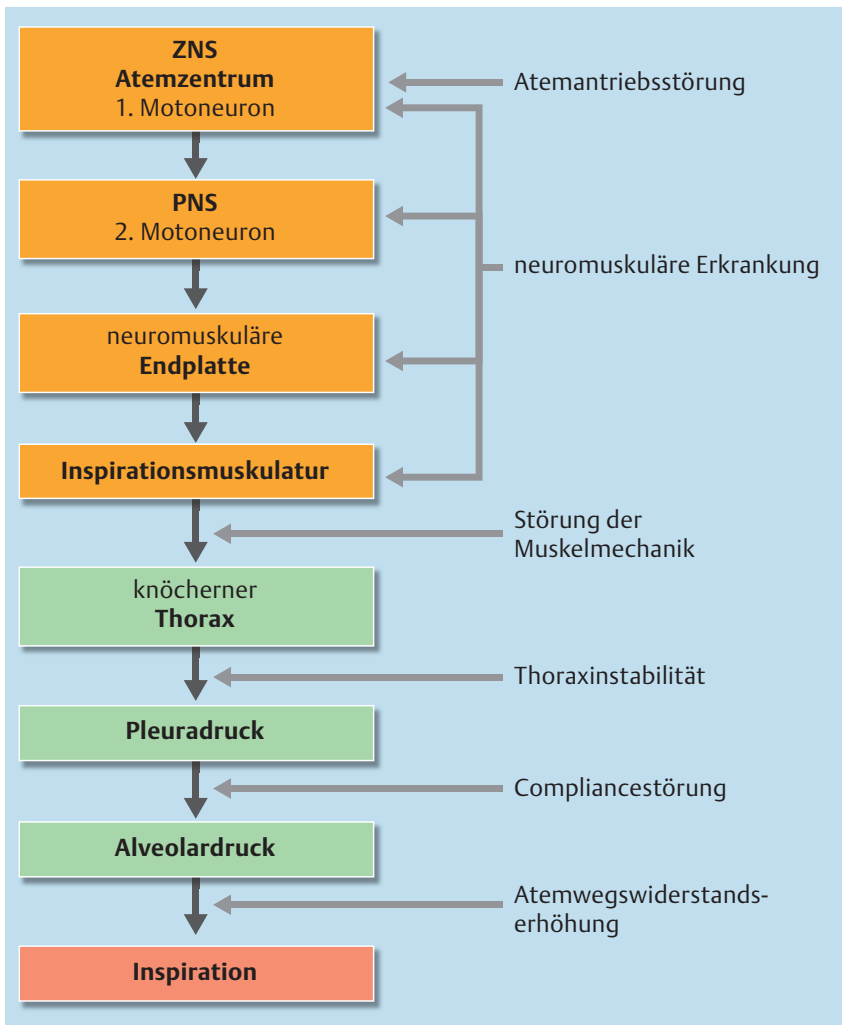

Abb. 1 Die Atempumpe sowie mögliche Lokalisationen bei deren Funktionsstörung. PNS: peripheres Nervensystem; ZNS: zentrales Nervensystem.

ge dieser Verfahren additiv zu verstehen sind, erlaubt erst die Kombination diverser Verfahren eine umfassende Diagnosestellung. So kann z.B. eine isolierte Zwerchfellschwäche bei Nervusphrenicus-Parese bei eher auf die globale Atemmuskelfunktion abzielenden Verfahren unerkannt bleiben [3]. Ferner gilt es zu beachten, dass erst die Kombination unterschiedlicher Testverfahren dazu in der Lage ist, die diagnostische Präzision zu erhöhen und die Menge an falsch positiven Befunden in Bezug auf eine mögliche Atemmuskelschwäche relevant zu reduzieren [6]. Eine erste Orientierung in Bezug auf die Atemmuskelfunktion liefern die mitarbeitsabhängigen, zumeist weniger komplexen und nicht-invasiven Verfahren. Wenn methodenspezifische Grenzwerte unterschritten werden, kann dies auf eine tatsächliche Atemmuskelschwäche hindeuten oder mitarbeitsbedingt eine solche vortäuschen. Der sichere Nachweis einer Atemmuskelschwäche kann dann nur durch den Einsatz von mitarbeitsunabhängigen Verfahren, welche zumeist einen komplexeren und teils invasiven Charakter aufweisen, gelingen [3-5,7].

Für die Ermittlung der Atemmuskelfunktion bei Kindern ergeben sich die folgenden Besonderheiten: Analog zur Lungenfunktion kann die Atemmuskelfunktion zumeist ab einem Alter von sechs Jahren zuverlässig gemessen werden. Die Mitarbeitsabhängigkeit der Messmethoden ist bei Kindern verständlicherweise noch ausgeprägter als bei Erwachsenen. Auch die Interpretation der mitarbeitsabhängigen Verfahren ist - wegen der hohen interindividuellen Variabilität - schwieriger als bei Erwachsenen. Die intra-individuelle Variabilität ist mit ca. 10\% akzeptabel und ermöglicht deshalb auch bei Kindern eine gute Verlaufsbeurteilung. 
Mitarbeitsabhänge Erfassung der Atemmuskelfunktion Der Mundverschlussdruck 0.1 Sekunden nach Inspirationsbeginn $\left[\mathrm{P}_{0.1}\right]$

Zur Bestimmung der Last hat sich der Mundverschlussdruck 100 msec nach Inspirationsbeginn $P_{0.1}$ bewährt. Hierbei wird zu Beginn einer Inspiration nach vorheriger normaler Exspiration während Ruheatmung ein Ventil vor dem Mund für $120 \mathrm{msec}$ verschlossen und der Munddruck $100 \mathrm{msec}$ nach Inspirationsbeginn bestimmt. Nach anschließender Öffnung des Ventils erfolgt der weitere Atemvorgang unbeeinflusst. Es sollen dabei 5-10 Verschlussmanöver in 1,5 Minuten durchgeführt werden. Wenn während des Manövers Atemnot auftritt, sollte dies in 2 Zyklen geschehen. Als Parameter der Last soll der Mittelwert oder besser noch der Median des $\mathrm{P}_{0.1}$ aus 5-6 Verschlüssen verwendet werden. Wenn der Mittelwert verwendet wird, müssen „Ausreißer“ (besonders hohe Drücke) eliminiert werden. Die Verschlüsse sollten unregelmäßig und zufällig erfolgen, um ein bewusstes oder unbewusstes Reagieren auf den Verschluss zu verhindern.

Der $\mathrm{P}_{0.1}$ ist ein Surrogatparameter für den mittleren Inspirationsdruck, der bei jedem Atemzug aufgebracht werden muss. Damit ist er einerseits ein Parameter der Last der Inspirationsmuskeln, andererseits aber auch ein Parameter des zentralen Atemantriebs. Der $\mathrm{P}_{0.1}$ hängt u.a. auch von der Druckübertragung von der Inspirationsmuskulatur zum Mund und vom Ausmaß der Ventilation ab, sodass diese Faktoren berücksichtigt werden müssen (s.u.).

Besonderheiten bei Kindern: Der zentrale Atemantrieb (und damit der $\mathrm{P}_{0.1}$ ) nimmt parallel zum spezifischen Grundumsatz und Sauerstoffverbrauch vom Neugeborenenalter bis zur Pubertät kontinuierlich ab. Der $\mathrm{P}_{0.1}$ von Mädchen und Jungen unterscheidet sich nicht. Bei Schulkindern ist ein $\mathrm{P}_{0.1}<0,45 \mathrm{kPa}$ nicht pathologisch [9].

\section{Durchfẗhrungsempfehlungen $\mathbf{P}_{0.1}[2,3,5]$ :}

- Auf Ruheatmung des Patienten achten

- Okklusion der Atemwege (inklusive Einsatz einer Nasenklemme) für mindestens $120 \mathrm{~ms}$

- Keine Vorankündigung der Okklusion, um Anpassungen des Atemmusters vor Registrierung des $\mathrm{P}_{0.1}$ zu vermeiden

- Registrierung des $\mathrm{P}_{0.1} 100 \mathrm{~ms}$ nach Inspirationsbeginn bei geschlossener Klappe (Verschlussdruck)

- Mindestens zwei Atemzüge trennen zwei $\mathrm{P}_{0.1}$-Messungen voneinander

- Registrierung des Median aus mindestens 5 Messungen

\section{Maximaler inspiratorischer/exspiratorischer}

Mundverschlussdruck: PImax/PEmax

Während einer maximalen in- respektive exspiratorischen Kraftentwicklung bei kurzzeitig am Munde verschlossenen Atemwegen erfolgt die Aufzeichnung des maximalen statischen in- bzw. exspiratorischen Mundverschlussdrucks (PImax bzw. PEmax). Hierbei muss ein Druckverlust über die Nasenöffnung durch Einsatz einer Nasenklemme vermieden werden. Diese Werte dienen zur Abschätzung der globalen Atemmuskelkraft und sind aufgrund der wenig komplexen Anforderungen an Patienten, Untersucher sowie Technik vergleichsweise weit verbreitet. Zudem sind die notwendigen technischen Voraussetzungen zur Messung bereits häufig z.B. in Bodyplethysmographen integriert oder als simple Handgeräte verfügbar. Von Vorteil ist hierbei, dass aufgrund der fehlenden intrathorakalen Volumenänderung bei iso- metrischer Atemmuskelkontraktion Störungen der Compliance bzw. Resistance des respiratorischen Systems ohne relevanten Einfluss bleiben. Um einen möglichen Verschluss der Glottis mit gestörter Druckübertragung bei einer rein statischen Druckentwicklung ebenso wie den Einsatz der buccalen Muskulatur mit falsch hohen Messwerten verhindern zu können, bedient man sich folgender Technik: Eine normierte Leckage in Form einer Kanüle (Länge: $4 \mathrm{~cm}$, Innendurchmesser: $1 \mathrm{~mm}$ ) garantiert einen minimalen Atemfluss [3,5].

Der PImax kann zum einen von der funktionellen Residualkapazität (FRC), zum anderen vom Residualvolumen (RV) aus registriert werden. Während bei der FRC-Registrierung die thorakalen Retraktionskräfte keine Beachtung finden müssen, addieren sich diese bei der Messung vom RV aus. Da vielen Patienten die statische Inspiration vom RV aus leichter gelingt und die Streuung der Messwerte keinen relevanten Unterschied aufweist [10], wird der PImax zumeist vom RV aus gemessen. Die Registrierung des PImax kann darüber hinaus entweder als Spitzen- oder als Plateauwert (über $1 \mathrm{~s}$ gehaltener PImax) erfasst werden. Obgleich im Ausland unüblich, erfolgt in Deutschland die Registrierung zumeist als Spitzenwert ( PImax $\left._{\text {peak }} \mathrm{RV}\right)[2,3,5,10]$.

Der PEmax wird als Spitzendruck von der totalen Lungenkapazität (TLC) aus erfasst. Leckagen am Mundstück, bedingt durch das positive Druckgefälle vom Mund zum Messgerät, müssen vermieden werden. Die buccale Muskulatur darf nicht zum Druckaufbau genutzt werden - dies sollte ausschließlich durch die exspiratorische Atemmuskulatur geschehen $[3,5]$.

Besonderheiten bei Kindern: Jungen generieren bereits vor der Pubertät einen höheren PImax und PEmax als Mädchen. Beide Werte korrelieren am besten mit dem Alter. Bei Jungen steigt der PImax von etwa $5 \mathrm{kPa}$ im Alter von 6 Jahren auf $9 \mathrm{kPa}$ im Alter von 16 Jahren und bei Mädchen von ca. 4,5 kPa auf 7,5 kPa [9].

Die 5\%-Perzentile des PImax liegt für Kinder von 6-16 Jahren zwischen 3 und 4 kPa; d. h. 95\% der untersuchten Kinder konnten einen höheren inspiratorischen Druck generieren. Ein PImax $>4 \mathrm{kPa}$ wird als nicht pathologisch eingestuft.

Altersabhängige Normwerte PImax bei Kindern[9] Jungen: $2,58+($ Alter $\times 0,39)[\mathrm{kPa}]$

Mädchen: 2,43+(Alter $\times 0,28)[\mathrm{kPa}]$

\section{Altersabhängige Normwerte PEmax bei Kindern [11] Jungen: $3,5+($ Alter $\times 5,5)[\mathrm{kPa}]$ \\ Mädchen 2,4+(Alter × 4,8) [kPa]}

Durchführungsempfehlungen PImax/PEmax [2-5, 10]:

- Maximaler Druckaufbau inspiratorisch (PImax-vom RV) bzw. exspiratorisch (PEmax - von der TLC) bei am Mundstück verschlossenen Atemwegen (inklusive Einsatz einer Nasenklemme)

- Motivation des Patienten durch den Untersucher zur Erbringung einer Maximalleistung

- Es sollten mindestens 5-7 Versuche durchgeführt und der höchste erzielte Wert registriert werden

- Fortsetzung der Messreihe, falls der finale Messwert den Maximalwert darstellt

- Die Differenz der beiden höchsten erzielten Maximalwerte sollte $<10 \%$ liegen

Z Zwei Einzelmessungen werden von einer Pause getrennt (ca. 15 - 30 Sekunden) 


\begin{tabular}{|c|c|c|c|}
\hline & & Frauen & Männer \\
\hline & & $\begin{array}{l}\text { nicht-pathologischer } \\
\text { Grenzwert }\end{array}$ & $\begin{array}{l}\text { nicht-pathologischer } \\
\text { Grenzwert }\end{array}$ \\
\hline$P_{0.1}$ & {$[\mathrm{kPa}]$} & $<0,3$ & $<0,3$ \\
\hline $\operatorname{PImax}_{1.0}$ RV & {$[\mathrm{kPa}]$} & $>6,0$ & $>7,0$ \\
\hline $\operatorname{PImax}_{\text {peak }}$ RV & {$[\mathrm{kPa}]$} & $>7,0$ & $>8,0$ \\
\hline PEmax & {$[\mathrm{kPa}]$} & $>7,0$ & $>10,0$ \\
\hline $\mathrm{P}_{0.1} / \mathrm{PImax}_{1.0} \mathrm{RV}$ & [\%] & $<4,5$ & $<4,5$ \\
\hline $\mathrm{P}_{0.1} / \mathrm{PImax}_{\text {peak }} \mathrm{RV}$ & [\%] & $<2,0$ & $<2,0$ \\
\hline $\mathrm{P}_{0.1}{ }^{*} \mathrm{t}_{\mathrm{i}} / \mathrm{V}_{\mathrm{T}}$ & {$\left[\mathrm{kPa}^{*} \mathrm{~s} / \mathrm{l}\right]$} & $<0,5$ & $<0,5$ \\
\hline Sn Pna & {$[\mathrm{kPa}]$} & $>6,0$ & $>7,0$ \\
\hline Sn Pdi & {$[\mathrm{kPa}]$} & $>8,0$ & $>10,0$ \\
\hline Tw Pmo & {$[\mathrm{kPa}]$} & $>1,0$ & $>1,0$ \\
\hline Tw Pdi & [kPa] & $>1,8$ & $>1,8$ \\
\hline Zwerchfellbewegung & {$[\mathrm{mm}]$} & $\geq 10,0$ & $\geq 10,0$ \\
\hline Zwerchfelldicke & {$[\mathrm{mm}]$} & $\geq 2,0$ & $\geq 2,0$ \\
\hline
\end{tabular}

Tab. 1 Nicht-pathologische Grenzwerte der diagnostischen Verfahren zur Messung der Atemmuskelfunktion [3].

$\mathrm{P}_{0.1}$ : Mundverschlussdruck nach 0.1 Sekunden Inspiration; PImax ${ }_{10}$ bzw. Plmax ${ }_{\text {peak }}$ RV: vom Residualvolumen aus bestimmter maximale inspiratorischer Mundverschlussdruck über 1.0 Sekunden bzw. Spitzenwert; PEmax: maximaler exspiratorischer Mundverschlussdruck; $\mathrm{P}_{0.1} /$ PImax: respiratorische Kapazität; $\mathrm{P}_{0.1}{ }^{*} \mathrm{t}_{\mathrm{i}} / \mathrm{V}_{\mathrm{T}}$ : spezifische inspiratorische Impedanz; Sn Pna: nasaler Sniff Druck; Sn Pdi: transdiaphragmaler Sniff Druck; Tw Pmo: Twitch Munddruck; Tw Pdi: transdiaphragmaler Twitch Druck.

\section{Respiratorische Kapazität: $\mathrm{P}_{0.1} /$ Plmax [Rechenwert]}

Bei Interpretation der Maximaldrücke als Ausdruck der maximalen Inspirationskraft muss das Lungenvolumen als Index der Länge der Inspirationsmuskulatur beachtet werden. Je höher das Lungenvolumen bei Bestimmung des maximalen Inspirationsdruckes ist, desto kürzer ist die Inspirationsmuskulatur und umso geringer ist der maximale Inspirationsdruck, obwohl keinerlei Kontraktionsstörung besteht. Somit muss beachtet werden, dass bei Patienten mit Lungenüberblähung geringere Inspirationsdrücke erreicht werden, obwohl die Kontraktilität der Inspirationsmuskulatur ungestört ist. Da sich aber das erhöhte Lungenvolumen sowohl bei der Inspirationsdruckentwicklung in Ruhe als auch bei der forcierten Inspiration gleichermaßen auswirkt, ist der Quotient $\mathrm{P}_{0.1} / \mathrm{PI}_{\max }$ als ein vom Lungenvolumen unabhängiger Parameter der Beanspruchung zu akzeptieren.

Eine Beanspruchung $\mathrm{P}_{0.1} / \mathrm{PI}_{\max }$ von über $4,5 \%$ bei Ruheatmung ist sicher erhöht. Der kritische Grenzwert bzgl. eines drohenden Atempumpversagens liegt bei rund 20 bis $25 \%[2,3]$.

\section{Effektive inspiratorische Impedanz: $\mathrm{P}_{0.1}{ }^{*} \mathrm{t}_{\mathrm{i}} / \mathrm{V}_{\mathrm{T}}$ [Rechenwert]}

Da der $\mathrm{P}_{01}$ bei Hyperventilation mit zunehmendem Atemminutenvolumen (VE) bzw. zunehmendem inspiratorischen Atemfluss $\left(\mathrm{V}_{\mathrm{T}} / \mathrm{t}_{\mathrm{i}}\right)$ ansteigt, werden unterschiedliche Atemminutenvolumen bzw. unterschiedliche Inspirationsgeschwindigkeiten bei Ruheatmung durch die Quotienten korrigiert, sodass die Quotienten weniger variieren als der Absolutwert des $\mathrm{P}_{0.1}$.

Multipliziert man den $P_{0.1}$ mit der Inspirationszeit $\left(t_{i}\right)$ und dividiert diesen Term durch das Tidalvolumen $\left(\mathrm{V}_{\mathrm{T}}\right)$, erhält man physikalisch das Korrelat eines Widerstandes (vgl. Einheit der Atemwegswiderstände). Anders gesagt: Der $\mathrm{P}_{0.1}$ entspricht dem notwendigen Druck, welcher in der Lage ist, einen definierten inspiratorischen Fluss $\left(\mathrm{V}_{\mathrm{T}} / \mathrm{t}_{\mathrm{i}}\right)$ aufzubauen. Die effektive inspiratorische Impedanz ist demnach ein Surrogat für die Last, welche der Inspirationsmuskulatur aktuell auferlegt ist. Dieser Term berücksichtigt - im Gegensatz zum $P_{0.1}$ - das Ausmaß der aktuellen Ventilation und kann daher die momentane atemmuskuläre Last abbilden [3]. Diese kann auch vereinfacht durch den Quotienten aus $\mathrm{P}_{0.1}$ und Minutenventilation $\left(\mathrm{V}_{\mathrm{E}}\right)$ abgeschätzt werden $\left(\mathrm{P}_{0.1} / \mathrm{V}_{\mathrm{E}}\right)$.

\section{Sniff Drücke: Sn P}

Die Sniff Drücke repräsentieren - ungleich der bisher abgehandelten Kenngrößen - dynamisch ermittelte Parameter, welche zudem eine höhere Zwerchfellspezifität aufweisen. Die Bezeichnung leitet sich von der Durchführung als kurzes, scharfes und maximales inspiratorisches Atemmanöver durch die Nase ab (engl. to sniff: durch die Nase einziehen, schniefen). Dieses physiologische Atemmanöver wird zumeist wesentlich besser toleriert als die (unphysiologischen) statischen Manöver (siehe oben). Auch die Standardabweichung der Messwerte fällt für die Sniff Drücke günstiger aus als z.B. für die PImax-Werte [3,5].

\section{Nasaler Sniff Druck: Sn Pna}

Der nicht-invasiv ermittelte nasale Sniff Druck (Sn Pna) repräsentiert einen verlässlichen Parameter zur Messung der inspiratorischen Atemmuskelkraft. Es muss jedoch beachtet werden, dass die tatsächliche inspiratorische Druckentwicklung unterschätzt wird, falls eine gestörte pleurale Druckübertragung vorliegt. Dies kann z.B. bei schwergradiger obstruktiver Lungenerkrankung (COPD) oder relevanten naso-pharyngealen bzw. laryngealen Pathologien der Fall sein [3,5].

\section{Transdiaphragmaler Sniff Druck: Sn Pdi}

Mit dem Ziel der Unabhängigkeit von einer gestörten pleuralen Druckübertragung kann die Registrierung der Sniff Drücke auch invasiv als so genannter transdiaphragmaler Sniff Druck (Sn Pdi) erfolgen. Transnasal applizierte Ballonkatheter erlauben die Messung der Druckdifferenz zwischen distalem Ösophagus und Magen (siehe auch Abschnitt „Twitch Drücke“) [3,5].

Besonderheiten bei Kindern: Aufgrund der einfachen Durchführung wird die Messung des Sniff Drucks von Kindern sehr gut toleriert; die intra- und inter-individuelle Variabilität ist geringer als beim PImax und auch atemmuskelschwache Kinder sind oft noch in der Lage reproduzierbare Sniff Manöver durchzuführen. Bei 180 Kindern im Alter von 6-17 Jahren betrug der Sn Pna $10,4 \pm 2,6 \mathrm{kPa}$ bei Jungen und 9,3 $\pm 2,3 \mathrm{kPa}$ bei Mädchen [12].

Die nicht-pathologischen Grenzwerte der jeweiligen mitarbeitsabhängigen Verfahren zur Messung der Atemmuskelfunktion (Ermittlung unter Einsatz eines Flansch-Mundstücks) sind in - Tab. 1 wiedergegeben. Grundlage sind publizierte Daten der 
Durchfïhrungsempfehlung Sn P [3-5]:

- Maximale, scharfe und kurze Inspiration (Dauer < $500 \mathrm{~ms}$ ) von der FRC bei geschlossenem Mund

- Motivation des Patienten durch den Untersucher zur Erbringung einer Maximalleistung

- Mindestens 5-7 Versuche und Registrierung des höchsten erzielten Wertes

- Fortsetzung der Messreihe, falls Maximalwert während finaler Messung vorliegt

- Die beiden höchsten erzielten Maximalwerte sollten < $10 \%$ differieren

- Trennung zweier Einzelmessungen durch eine Pause (ca. 15 - 30 Sekunden)

einzelnen Verfahren sowie Einschätzungen der Expertengremien $[2-5,7,10,13]$.

\section{Hustenspitzenfluss (engl. Peak Cough Flow): PCF}

Die Atemmuskulatur hat eine wesentliche Bedeutung für die Hustenfunktion, wobei durch eine Schwäche von Inspirations-, Exspirations- oder Glottismuskulatur bzw. einer Kombination aus diesen - insbesondere bei neuromuskulären Erkrankungen - eine Hustenschwäche resultieren kann. Diagnostisch kommt hier neben der Messung des PEmax (siehe oben) die Erfassung des Hustenspitzenflusses in Betracht. Dieser kann durch Husten in ein handelsübliches „Peakflowmeter“ (wie es z. B. zur Asthma-Kontrolle eingesetzt wird) einfach bestimmt werden, wobei auch hier eine Nasenklemme verwendet werden sollte. PCF-Werte von $<2701 / \mathrm{min}$ gelten als pathologisch für Erwachsene [14]. Bei Kindern und Jugendlichen ist ein $\mathrm{PCF}<160 \mathrm{l} / \mathrm{min}$ mit dem gehäuften Auftreten von Pneumonien verbunden, während Werte von $>200$ l/min negativ prädiktiv sind [15].

\section{Mitarbeitsunabhängige Erfassung der Atemmuskel- funktion}

Sämtliche oben abgehandelte Verfahren zur Messung der Atemmuskelfunktion sind in hohem Maße von der Kooperation und Mitarbeit des Patienten abhängig. Während hierbei (hoch-)normale Werte eine klinisch relevante Atemmuskelschwäche mit hoher Verlässlichkeit ausschließen können, bereiten Werte unterhalb eines nicht-pathologischen Grenzwertes Schwierigkeiten: So bleibt hierbei unklar, ob mitarbeitsbedingt falsch-niedrige Werte registriert wurden oder ob tatsächlich eine manifeste Schwäche der Atemmuskulatur vorliegt. Die im Folgenden diskutierten mitarbeitsunabhängigen Verfahren sind in der Lage, diese Befunde zu objektivieren [3].

\section{Twitch Drücke: Tw P}

Den aktuellen Goldstandard in der Diagnostik der Atemmuskelfunktion bilden Druckaufzeichnungen nach elektro-magnetischer (früher: elektrischer) Stimulation des Nervus phrenicus. Via kurzzeitigem (wenige ms) Stromfluss im Nervus phrenicus nach Erzeugung eines hochenergetischen Magnetfeldes wird eine singuläre Zwerchfellkontraktion herbeigeführt. Diese kurzzeitige „Zuckung“ der innervierten Muskeln führte zur Namensgebung des Verfahrens (engl. to twitch: zucken). Als zuverlässigste Stimulationsart hat sich die bilaterale anteriore Magnetstimulation des Nervus phrenicus (BAMPS) herausgestellt. Die Platzierung der Stimulationsspulen erfolgt hierbei am dorsalen Rand der Mm. sternocleidomastoidei auf Höhe der Cartilago cricoidea

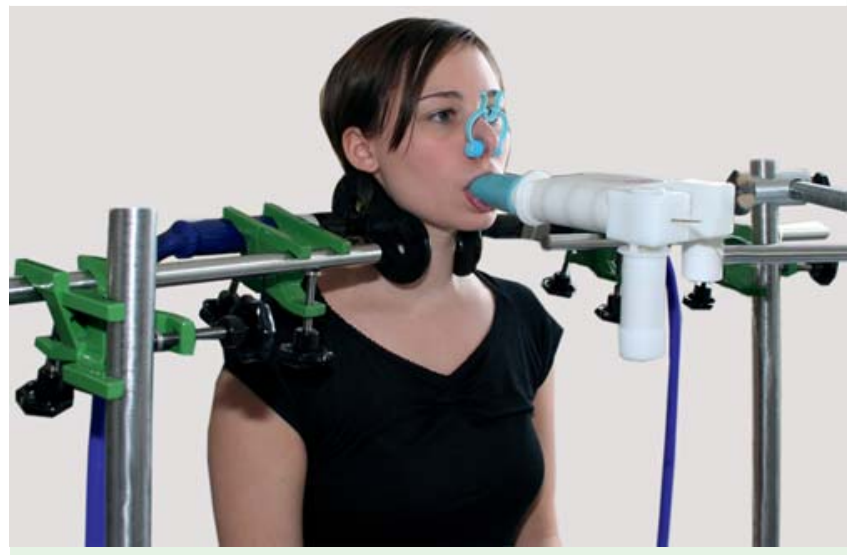

Abb.2 Messanordnung bei bilateraler anteriorer Magnetstimulation des Nervus phrenicus (BAMPS) [3]. Spulenplatzierung: Hinterrand der Mm. sternocleidomastoidei auf Höhe der Cartilago cricoidea. Neben dem Twitch Munddruck lässt sich bei unveränderter Messanordnung nach Applikation von Ballonkathetern auch der transdiaphragmale Twitch Druck registrieren.

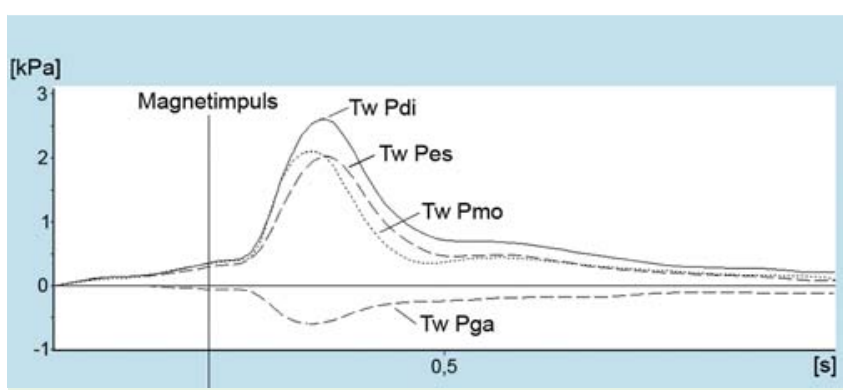

Abb.3 Druck-Zeit-Kurven für die Twitch Drücke bei bilateraler anteriorer Magnetstimulation des Nervus phrenicus (BAMPS; inspiratorischer, druckgesteuerter Trigger) [3]. Tw Pdi: transdiaphragmaler Twitch Druck; Tw Pes: ösophagealer Twitch Druck; Tw Pga: gastraler Twitch Druck; Tw Pmo: Twitch Munddruck.

$[13,16,17]$. Das sichere Erreichen einer so genannten „Supramaximalität" der Stimulation ist essentiell: Nur in diesem Fall würde eine weitere Erhöhung der Impulsstärke nicht in einer weiteren Erhöhung der Druckantwort resultieren (da sämtliche motorischen Einheiten innerviert wurden) [3]. In $\bullet$ Abb. 2 ist die Versuchsanordnung bei BAMPS wiedergegeben.

End-exspiratorisch beträgt der transdiaphragmale Druck bei Ruheatmung per definitionem $0 \mathrm{kPa}$ [18]. Ferner steigt der registrierte Twitch Druck linear mit abnehmendem Lungenvolumen in Richtung des RV (zunehmende Vordehnung der Muskelfasern) $[19,20]$. Aus diesen Gründen muss sichergestellt werden, dass der Twitch Druck bei der FRC gemessen wird, und die Einhaltung dessen sollte überprüft werden [13,17]. Wird ein Muskel stark innerviert, so ist kurze Zeit später seine Kontraktilität erhöht (so genannte „Twitch Potentiation“). Dies muss entsprechende Berücksichtigung finden (z.B. durch eine standardisierte Ruhepause vor Messung oder durch vorherige maximale willentliche Kontraktionen) [3]. Exemplarische Druck-Zeit-Kurven für die Twitch Drücke sind in $\mathbf{A b b} . \mathbf{3}$ wiedergegeben. 


\section{Twitch Munddruck: Tw Pmo}

Grundvoraussetzung der adäquaten Messung des nicht-invasiven Twitch Munddrucks (Tw Pmo) ist die ungestörte Druckübertragung von der Pleura an den Mund. Erfolgt die Stimulation endexspiratorisch in Atemruhelage (i.e. FRC), kann ein inkonsistenter Verschluss der Glottis die Validität der Messung gefährden. Daher wird ein so genannter „Triggermechanismus“ eingesetzt: Erst nach Erreichen eines minimalen Atemflusses bzw. Atemwegsdrucks durch das oben genannte Leckagesystem erfolgt die automatisierte Applikation des Magnetimpulses. Am zuverlässigsten hat sich hierbei ein druckgesteuerter inspiratorischer Triggermechanismus erwiesen $[3,13,17]$.

Transdiaphragmaler Twitch Druck: Tw Pdi

Der invasiv zu ermittelnde transdiaphragmale Twitch Druck (Tw Pdi) erfordert die transnasale Einlage von Ballonkathetern in den Magen und den distalen Ösophagus $[4,13]$. Aus einer Punkt-zu-Punkt-Subtraktion der gastralen (TwPga, ca. 1/3 des Tw Pdi) von den ösophagealen (TwPes, ca. 2/3 des Tw Pdi) Druckwerten errechnet sich der Tw Pdi gemäß folgender Gleichung (1) [3].

TwPdi [kPa]=TwPes [kPa] - TwPga [kPa] (Gl. 1)

Unter Beachtung der jeweiligen Druck-Dehnungs-Kurven erfolgt eine standardisierte Luftbefüllung der Ballons (näherungsweise etwa $1,0 \mathrm{ml}$ für den ösophagealen und etwa 2,0 ml für den gastralen Ballon [21]). Nur hierdurch lässt sich eine adäquate Druckübertragung gewährleisten. Aufgrund der Tatsache, dass der Öffnungszustand der Glottis die Druckübertragung von der Pleura auf Ösophagus und Magen nicht beeinflusst, erfordert die Registrierung des Tw Pdi keinen Triggermechanismus [3].

\section{Durchfïhrungsempfehlungen Tw $P[3,5]$ :}

- Überprüfung Kontraindikationen (Magnetfeld): u.a. Herzschrittmacher sowie im Kopf-Hals-Bereich befindliche elektronische und metallene Implantate; Schwangerschaft

- Platzierung der Ballonkatheter (nur bei Tw Pdi-Messung): Transnasaler Vorschub beider Ballons in den Magen Rückzug des proximalen Ballons bis ösophago-gastraler Übergang (Druckumkehr)

Rückzug um weitere $10 \mathrm{~cm}$ und anschließende Fixierung Überprüfung der korrekten Katheterlage anhand „SniffManöver" (siehe oben)

- 15-20 Minuten Ruhepause ohne übermäßige Zwerchfellbeanspruchung (z. B. lautes Sprechen oder körperliche Belastung) bzw. vorsätzliche Potenzierungsmanöver (PImax-Manöver) aufgrund „Twitch Potentiation“

- Zwei Einzelmessungen werden durch eine Pause getrennt (ca. 30 Sekunden)

- Registrierung des Mittelwerts aus 5 Messungen (da mitarbeitsunabhängig)

\section{Elektromyografie}

Die Elektromyografie (EMG) der Atemmuskulatur erlaubt - teils in Kombination mit der elektro-magnetischen Nervenstimulation - eine detaillierte Analyse der peripheren und der zentralen nervalen Anteile der Atempumpe, des neuro-muskulären Übergangs sowie spezifischer Muskelerkrankungen. Die Ableitung kann invasiv (Nadelelektroden, Ösophagus-EMG) oder nicht-invasiv (Klebeelektroden) erfolgen. EMG-Messungen unterliegen zahlreichen Störfaktoren wie z.B. durch die Elektrodenlokalisation, -charakteristik, Umgebungsrauschen, cross-talk benachbarter Muskulatur und Bewegungsartefakte [22]

Das EMG der Atemmuskulatur kann an der Atemhilfsmuskulatur und dem Zwerchfell abgeleitet werden. Die sogenannte „parasternale Muskulatur“ (2. Interkostalraum parallel des Sternums) stellt einen einfach zugänglichen Messpunkt dar, welcher in klinischen Studien mehrfach validiert wurde. Das Zwerchfell kann an mehreren Oberflächenlokalisationen abgeleitet werden, unterliegt jedoch einer hohen Störanfälligkeit. Als robuster Ableiteort hat sich die Positionierung der ableitenden Elektrode $5 \mathrm{~cm}$ oberhalb des Xiphoids und der Referenzelektrode in einem Radius von $16 \mathrm{~cm}$ am rechten unteren Rippenbogen erwiesen [23]. Durch die EMG wird das Überlagerungssignal der einzelnen Aktionspotenziale dargestellt (CMAP; engl. compound muscle action potential). Neben Erfassung der Dauer bis zur Signalantwort auf eine Stimulation sowie der Amplitude erfolgt zumeist auch eine mathematische Verarbeitung des Signals. Bei Registrierung von Spontanatmung bzw. forcierter Atmung erfolgt dies in einem definierten Aktivierungszeitfenster (z.B. $200 \mathrm{~ms}$ ) um das Aktivitätsmaximum. Hier hat sich die sogenannte „Root-MeanSquare“-Berechnung etabliert, welche auch als Parameter für die globale Muskelfaseraktivierung dienen kann. Es ist zu beachten, dass RMS-Werte nicht inter-individuell verglichen werden können. Der Quotient aus dem RMS-Wert während Ruheatmung und maximaler Aktivierung (willkürlich und/oder durch Stimulation) erlaubt Aussagen bezüglich des Ausmaßes des zentralen Atemantriebs und erlaubt somit auch den inter-individuellen Vergleich von EMG-Daten.

Normwerte hinsichtlich der Nervenleitgeschwindigkeit des $\mathrm{N}$. phrenicus sind abhängig von Stimulationstechnik sowie dem Stimulationsort und der Ableiteart, sodass an dieser Stelle auf weiterführende Literatur verwiesen wird [24].

\section{Bildgebende Verfahren}

Von den bildgebenden Verfahren wird hier aufgrund der Komplexität bzw. Fachzugehörigkeit nicht auf die Magnetresonanztomografie sowie auf Techniken eingegangen, welche ionisierende Strahlung zur Anwendung bringen (z.B. Röntgen-Aufnahmen inkl. Durchleuchtung, Computertomografie). Gleichwohl können diese Verfahren teils wertvolle Informationen zur Atemmuskelfunktion liefern.

Eine vergleichsweise wenig komplexe, nicht-invasive und zudem kosteneffiziente Möglichkeit der Untersuchung der Atemmuskelfunktion bietet die Ultraschalluntersuchung des Diaphragmas. Hierbei lässt sich die Auslenkung des Zwerchfells beurteilen, wobei Werte $<10 \mathrm{~mm}$ als Zwerchfelldysfunktion gewertet werden [25]. Dieses Verfahren kann z. B. bei langzeitbeatmeten Patienten auch bettseitig angewendet werden und besitzt einen ähnlichen prädiktiven Wert im Hinblick auf ein mögliches Weaning-Versagen wie der klinisch etablierte „Rapid-Shallow-Breathing-Index“ (Verhältnis von Atemfrequenz zu Atemzugvolumen) [25]. Die - Abb.4 illustriert die sonografische Messung der Zwerchfellexkursion an einem gesunden Probanden. 


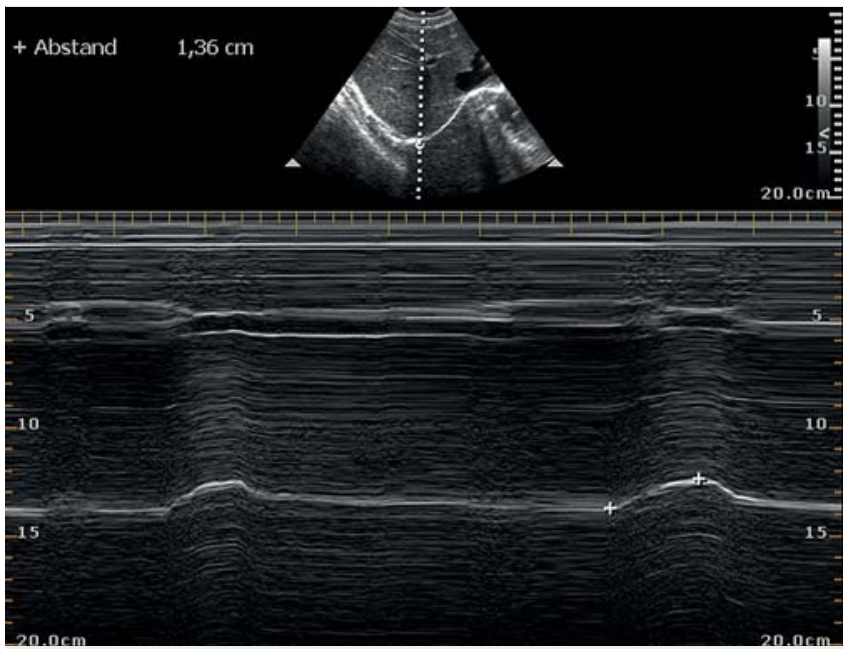

Abb.4 Sonografisch ermittelte Zwerchfellbeweglichkeit [28]. M-Mode während Ruheatmung im Liegen. Die Zwerchfellexkursion (weiße Linie: diaphragmale Pleura) beträgt $\geq 10 \mathrm{~mm}$ (i. e. kein Hinweis auf diaphragmale Dysfunktion). Anschallwinkel $\geq 70^{\circ}$ unmittelbar unterhalb des Xiphoids (Schallfenster: Leber).

Zusätzlich kann sonografisch die Zwerchfelldicke ermittelt werden und so z.B. im Rahmen einer invasiven Beatmungstherapie deren mögliche Abnahme kontrolliert werden [26]. Exemplarisch zeigt Abb.5 die Ausmessung der Zwerchfelldicke bei einem gesunden Probanden.

Sämtliche hier vorgestellte Verfahren zur Messung der Atemmuskelfunktion sind mit den jeweiligen Charakteristika in - Tab. 2 nochmals zusammengefasst [3].

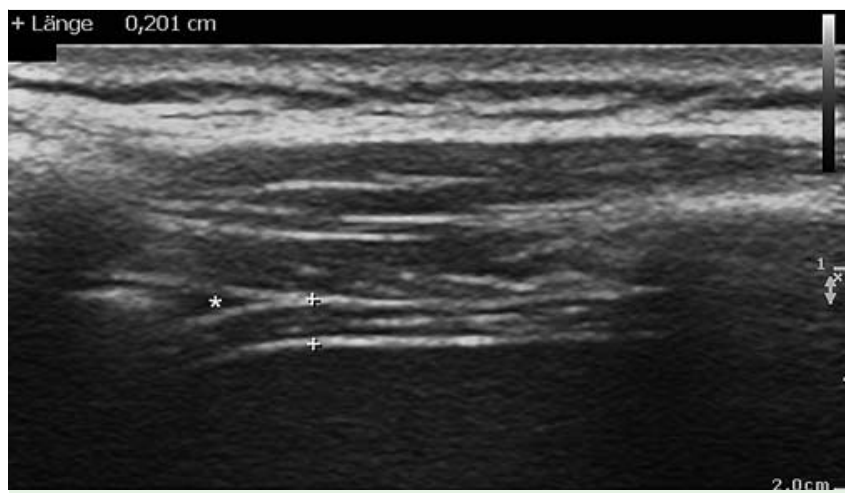

Abb.5 Sonografisch ermittelte Zwerchfelldicke [28]. Zweidimensionaler Echtzeitmodus mit end-exspiratorischer Dicke des Zwerchfells rechts. Schallfenster: mittlere Axillarlinie auf Höhe der diaphragmalen AppositionsZone. Messpunkte: diaphragmale und peritoneale Pleuralinie. Stern-Markierung $\left({ }^{*}\right)$ : Recessus costodiaphragmaticus.

\section{Durchführungsempfehlung Zwerchfellultraschall [25-27]:}

- Den Patienten in liegende Körperposition bringen

- Zeit-Bewegungs-Modus („(T)M-Mode“) wählen und Hemidiaphragma Exkursionen in mm rechts und links über die unteren Interkostalräume bzw. unterhalb des Xiphoids messen (Schallfenster: Leber bzw. Milz)

- Schallwinkel $\geq 70^{\circ}$ beachten

- Zur Messung der Zwerchfelldicke Helligkeits-modulierenden Modus („B-Mode“) bzw. zweidimensionalen Echtzeitmodus wählen

- Einstellung der Appositions-Zone des Zwerchfells in der mittleren Axillarlinie

- Messung end-exspiratorisch an der dicksten Stelle zwischen diaphragmaler und peritonealer Pleuralinie

Tab.2 Kenngrößen der Messverfahren zur Diagnostik der Atemmuskelfunktion [3].

\begin{tabular}{|c|c|c|c|c|c|c|}
\hline & $\begin{array}{l}\text { mitarbeits- } \\
\text { abhängig }\end{array}$ & $\begin{array}{l}\text { invasive } \\
\text { Messung }\end{array}$ & $\begin{array}{l}\text { technischer } \\
\text { Aufwand }\end{array}$ & $\begin{array}{l}\text { Reproduzierbarkeit } \\
\text { der Messung }\end{array}$ & $\begin{array}{l}\text { Streuung der } \\
\text { Normwerte }\end{array}$ & $\begin{array}{l}\text { Datenlage zu den } \\
\text { Normwerten }\end{array}$ \\
\hline$P_{0.1}$ & ja & nein & $\downarrow$ & $\uparrow$ & $\leftrightarrow$ & hervorragend \\
\hline PImax & ja & nein & $\leftrightarrow$ & $\leftrightarrow$ & $\uparrow$ & hervorragend \\
\hline PEmax & ja & nein & $\leftrightarrow$ & $\downarrow$ & $\uparrow$ & ausreichend \\
\hline $\mathrm{P}_{0.1} / \mathrm{PImax}$ & ja & nein & $\leftrightarrow$ & $\leftrightarrow$ & $\uparrow$ & ausreichend \\
\hline $\mathrm{P}_{0.1}{ }^{*}$ ti/VT & ja & nein & $\downarrow$ & $\uparrow$ & $\leftrightarrow$ & unzureichend \\
\hline Sn Pna & ja & nein & $\leftrightarrow$ & $\uparrow \uparrow$ & $\downarrow \downarrow$ & ausreichend \\
\hline Sn Pdi & ja & ja & $\uparrow \uparrow$ & $\uparrow \uparrow$ & $\downarrow \downarrow$ & ausreichend \\
\hline Tw Pmo & nein & nein & $\uparrow$ & $\uparrow \uparrow$ & $\downarrow$ & unzureichend \\
\hline Tw Pdi & nein & ja & $\uparrow \uparrow$ & $\uparrow \uparrow$ & $\downarrow$ & unzureichend \\
\hline Ultraschall & nein & nein & $\uparrow$ & $\uparrow \uparrow$ & $\leftrightarrow$ & unzureichend \\
\hline EMG & nein & nein & $\uparrow \uparrow$ & $\uparrow$ & $\uparrow$ & unzureichend \\
\hline
\end{tabular}

EMG: Elektromyografie (Oberfläche); $P_{0.1}$ : Mundverschlussdruck nach 0.1 Sekunden Inspiration; PImax: maximaler inspiratorischer Mundverschlussdruck; PEmax: maximaler exspiratorischer Mundverschlussdruck; $\mathrm{P}_{0.1} /$ PImax: respiratorische Kapazität; $\mathrm{P}_{0.1}{ }^{*} \mathrm{t}_{\mathrm{i}} / \mathrm{V}_{\mathrm{T}}$ : spezifische inspiratorische Impedanz; Sn Pna: nasaler Sniff Druck; Sn Pdi: transdiaphragmaler Sniff Druck; Tw Pmo: Twitch Munddruck; Tw Pdi: transdiaphragmaler Twitch Druck.

\section{Interessenkonflikt}

Die Autoren geben an, dass kein Interessenkonflikt besteht. 


\section{Literatur}

1 Criée C-P, Laier-Groeneveld G. Die Atempumpe: Atemmuskulatur und intermittierende Selbstbeatmung. Thieme; 1995

2 Criée CP. Empfehlungen der Deutschen Atemwegsliga zur Messung der inspiratorischen Muskelfunktion. Pneumologie 2003; 57: 98 - 100

3 Kabitz H-J, Windisch W. Diagnostik der Atemmuskelfunktion: State of the Art. Pneumologie 2007; 61: $582-587$

4 Moxham J. Respiratory Muscles. In: Hughes JMB, Pride NB (Hrsg). Lung Function Tests. 1. Aufl. WB Saunders; 1999: 57-72

5 American Thoracic Society/European Respiratory Society ATS/ERS. Statement on respiratory muscle testing. Am J Respir Crit Care Med 2002; 166: $518-624$

6 Steier J, Kaul S, Seymour J et al. The value of multiple tests of respiratory muscle strength. Thorax 2007; 62: 975-980

7 Polkey MI, Green M, Moxham J. Measurement of respiratory muscle strength. Thorax 1995; 50: 1131 -1135

8 Whitelaw WA, Derenne JP. Airway occlusion pressure. J Appl Physiol 1993; 74: 1475 - 1483

9 Mellies $U$, Schultze S, Schwake C et al. Respiratory muscle function in 300 healthy children. Eur Resp J 2001; 18: P827 [Abstract].

10 Windisch $W$, Hennings $E$, Sorichter $S$ et al. Peak or plateau maximal inspiratory mouth pressure: which is best? Eur Resp J 2004; 23: 708 713

11 Wilson SH, Cooke NT, Edwards RH et al. Predicted normal values for maximal respiratory pressures in caucasian adults and children. Thorax 1984; 39: $535-538$

12 Stefanutti D, Fitting JW. Sniff nasal inspiratory pressure. Reference values in Caucasian children. Am J Respir Crit Care Med 1999; 159: 107 111

13 Kabitz H-J, Walker D, Walterspacher $S$ et al. Controlled twitch mouth pressure reliably predicts twitch esophageal pressure. Respir Physiol Neurobiol 2007; 156: $276-282$

14 Bach JR, Ishikawa Y, Kim H. Prevention of pulmonary morbidity for patients with Duchenne muscular dystrophy. Chest 1997; 112: $1024-1028$
15 Dohna-Schwake C, Ragette R, Teschler $H$ et al. Predictors of severe chest infections in pediatric neuromuscular disorders. Neuromuscular disorders 2006; 16: 325 - 328

16 Mills GH, Kyroussis D, Hamnegard CH et al. Bilateral magnetic stimulation of the phrenic nerves from an anterolateral approach. Am J Respir Crit Care Med 1996; 154: 1099-1105

17 Windisch W, Kabitz H-J, Sorichter S. Influence of different trigger techniques on twitch mouth pressure during bilateral anterior magnetic phrenic nerve stimulation. Chest 2005; 128: 190-195

18 Thews G. Lungenatmung. In: Schmidt RF, Thews G (Hrsg.), Physiologie des Menschen. 27. Aufl. Springer; 1997: 565-591

19 Polkey MI, Hamnegård CH, Hughes PD et al. Influence of acute lung volume change on contractile properties of human diaphragm. J Appl Physiol 1998; 85: $1322-1328$

20 Walker DJ, Walterspacher S, Schlager D et al. Characteristics of diaphragmatic fatigue during exhaustive exercise until task failure. Respir Physiol Neurobiol 2011; 176: 14-20

21 Milic-Emili J, Mead J, Turner JM et al. Improved technique for estimating pleural pressure from esophageal balloons. J Appl Physiol 1964; 19: 207-211

22 Bolton CF. AAEM minimonograph \#40: clinical neurophysiology of the respiratory system. Muscle \& nerve 1993; 16: 809-818

23 Dionne A, Parkes A, Engler B et al. Determination of the best electrode position for recording of the diaphragm compound muscle action potential. Muscle Nerve 2009; 40: 37-41

24 Bolton CF, Chen R, Wijdicks EFM et al. Neurology of breathing. Elsevier; 2004

25 Kim WY, Suh HJ, Hong S-B et al. Diaphragm dysfunction assessed by ultrasonography: influence on weaning from mechanical ventilation. Crit Care Med 2011; 39: 2627-2630

26 Grosu HB, Lee YI, Lee J et al. Diaphragm muscle thinning in patients who are mechanically ventilated. Chest 2012; 142: 1455-1460

27 Nason LK, Walker CM, McNeeley MF et al. Imaging of the diaphragm: anatomy and function. Radiographics 2012; 32: E51-E70

28 Kabitz H-J, Windisch W, Schönhofer B. Ventilator induzierter Zwerchfellschaden: ein Update. Pneumologie 2013; 67: 435-441 